\title{
An Approach to Exudates Detection using Color Reference Segmentation in Retinal Fundus Image
}

\author{
Diana Tri Susetianingtias \\ Gunadarma University \\ Margonda Raya Street 100 \\ Depok - Indonesia
}

\author{
Sarifuddin Madenda \\ Gunadarma University \\ Margonda Raya Street 100 \\ Depok - Indonesia
}

\author{
Rodiah \\ Gunadarma University \\ Margonda Raya Street 100 \\ Depok - Indonesia
}

\begin{abstract}
The result of retinal images from fundus cameras are often provide unclear blood vessel of retinal images. As consequent, the ophthalmologists find it difficult to analyze the retinal images. Fundus images analyses require a longer time to achieve the test result especially for the patient with diabetic retinopathy. The research conducted in this paper introducing a space color reference approach to perform exudates segmentation. Exudates are one of the symptoms that cause diabetic retinopathy. The existence of exudates can be characterized by the appearance of fundus image in yellowish color with varying size and shape. The difficulties in exudates detection are due to the similar color intensity with optic disc (retinal blind spot), but with smaller size compare to optic disc. The study in this paper proposes color space approach where the object of interest area is used as exudates color references for retinal segmentation. The results of the experiments show that exudates detection based on color space reference are successfully segmented where the optic disc is not segmented as a part of exudates.
\end{abstract}

\section{Keywords}

Exudates, Fundus Image, Region of Interest, RGB Color Space, Segmentation

\section{INTRODUCTION}

Retina is an important part of the vision that has light stimulus receptor receiver. It carries visual information before transmitted to visual cortex through the optical nerve [1]. In a diabetic retinopathy there is some decay that occurs gradually (i.e., retinal muscular damage, the leak of optic disc layer). Those kinds of conditions, eventually can lead blurred vision and even blindness. If the retinal damage is severe, a patient with diabetic can become permanently visual impaired even if an attempt is made. Diabetic retinopathy is divided into two types namely the Non-Proliferation of diabetic retinopathy (NPDR) and proliferation diabetic retinopathy (PDR). In the more common form of NPDR, a new vein do not grow (evolve) in the wall of the vein in a weakened retina. Moreover a small protrusions (microaneurysm) protruding from the wall of the smaller vein and sometimes fluid as well as blood leaked into the retina. A large retinal blood vessel can swell and its diameter becoming irregular. NPDR can progress from mild to a severe state since many blood vessel are clogged. One way to detect the existence of diabetic retinopathies is by the emergence of the exudates. Exudates appear when there are lipid or fat that leaked on the abnormal blood vessel or aneurysms[2].

Based on visual observation of an ophthalmologist, the exudates appear in a yellowish white color with different size, shape and location. The exudates sometime can be seen as an individual form or in the form of cluster. This research carried out by localized the existence of exudates as one characteristic of diabetic retinopathy. K-Mean Clustering algorithm was employed to classify the segmentation of retinal fundus images based on the image's color and texture. CIELAB color models are used since the components integrity and component color are apart. It consists of $L$ components which stated that the brightness and color components for $a$ and $b$ are based on coordinate color space CIE XYZ. Therefore, by transforming the RGB color model that does not provide any information about integrity or brightness to a model that give integrity information, the expected image input will have a varied intensity[3]. This study utilized GLCM method to determine the exudates based on its textures using grey value histogram by calculating the degree of contrast, granularity and the roughness of an area adjacency between pixels inside the image fundus. The proposed approach used varied algorithms to identify and to segment exudates detection as one feature of diabetic retinopathy. The selected feature vector will be classified inside the exudate and non-exudate using Support Vector Machine (SVM) [4]. Likewise, the study for exudates detection can be done using green channel compositions on retinal image fundus. The study in this paper using green channel since it has the exact exudates composition. On the contrast with red channel which oversaturated or blue channel that under saturated. Many researchers conducted experiments with green channel composition for image processing, because the saturation of green channel located in exact composition of exudates. Images with low contrast level have a narrow histogram component and located in the middle of grey level. In grey scale images, lower contrast level can create the image object look blur. On the contrary, on high level image contrast, the histogram components are evenly spread through the range of grey level scale. Exudate detection with green channel composition resulting morphological original exudates image changes [5]. A Contrast Limited Adaptive Histogram Equalization method is used in [6] to improve the contrast of fundus image that operated on smaller region by implementing bilinear interpolation. As a result, the unseen neighborhood edges of smaller regions in retinal blood vessel can be detected. A green channel of retinal fundus images is implemented then the next step is to restore the image contrast by exploiting the contrast limited adaptive histogram equalization. The segmentation of fundus retinal blood vessel image required thresholds which are based on the value of pixels intensity. This segmentation carried out by assuming the image intensity can be divided into two non overlapping groups. The first group is based on object and the second one is based on the background. Both of these groups are built upon the threshold values $T$ on histogram. The accuracy of cut-points image detection shows that the selection of image contrast in the process of image enhancement will have some effect on the ground truth level of cut-point image detection. The segmentation of the blood vessel becoming lesser due to 
the value of the image contrast that is too small. On the contrary, when the contrast of the image values are enormous, the pixels of blood vessel that are detected also massive. When the blood vessel that being segmented is small, the detection of image cut-points becoming minor as well, thus the accuracy is less. On the other side, when the values of the image contrasts are too huge, the errors of image cut-points detection becoming high thus the obtained accuracy values are small as well [6]. The research conducted by [7] employed a feature extraction using Scale Invariant Feature Transform (SIFT) with $13 \times 13$ and $25 \times 25$ ROI fundus image windows size. The Gaussian Support Vector Machine (SVM) is used in their study to classify each patch and to obtain the probability of each batch that contain the exudates. The study in their paper are utilized the median filter for the first channel of the HIS image. Then for the next step, they are using Contrastlimited Adaptive Histogram Equalization methods. This method is done before applying the method of Otsu Thresholding. The last step is eliminating the optical disc.

In this paper, the exudates segmentation on the area of image fundus based on color references is proposed. Based on visual observation of an ophthalmologist, exudates appear in a yellowish white color with different size, shape and location. This research is expected to detect the exudates quickly and accurately. The colors in exudates can be used as references for diabetic retinopathy segmentation.

\section{MATERIALS AND METHODS}

The experiment in this paper utilized the image fundus from STARE database. The Ophthalmoscope used camera fundus TopCon TRV-50 with the angle $35^{\circ}$ FOV. The size of the image is $700 \times 605$ pixels.

\subsection{Determining ROI Exudates}

The exudates sometime can be seen as individual fundus or in the form of clusters with yellowish white color. To perform exudates area segmentation on fundus image, semi-automatic segmentation on exudates area as Region of Interest is carried out. The steps of the algorithms can be seen below [8]:

1. Read the fundus image (Automatically the image is stored in 3D RGB matrix).

2. Determine the color pixel of the object of interest area as exudates color reference for segmentation.

3. Determine the range of threshold distance color.

4. When the color that are used for the experiments are other than the one in RGB color space, convert the image and reference pixel color from RGB space color to a color space used for segmentation.

5. For each pixel in fundus image, the color distance towards reference color are computed with these following stages:

a. Compute the distance between two colors using distance formula in equation 1 and 2 [8] with exudates color space.

$$
\begin{aligned}
& \Delta E=\sqrt{\left(R_{1}-R_{2}\right)^{2}+\left(G_{1}-G_{2}\right)^{2}+\left(B_{1}-B_{2}\right)^{2}} \\
& \Delta E=\left|R_{1}-R_{2}\right|+\left|G_{1}-G_{2}\right|+\left|B_{1}-B_{2}\right|
\end{aligned}
$$

The color distances between two pixels are calculated using city block distance, which subsequently expressed in equation (1) and (2) where: $\left(R_{1}, G, B_{1}\right)$ and $\left(R_{2}, G_{2}, B_{2}\right)$ are stands for Red, Green and Blue color component. b. If the color distance value is less than threshold value, the pixel is the component of the segmented exudates area. Since the pixel is not part of area segmentation, therefore this area is removed.

In general, the semi-automatic segmentation algorithm towards exudates area as Region of Interest (ROI) can be seen in Figure 1 below.

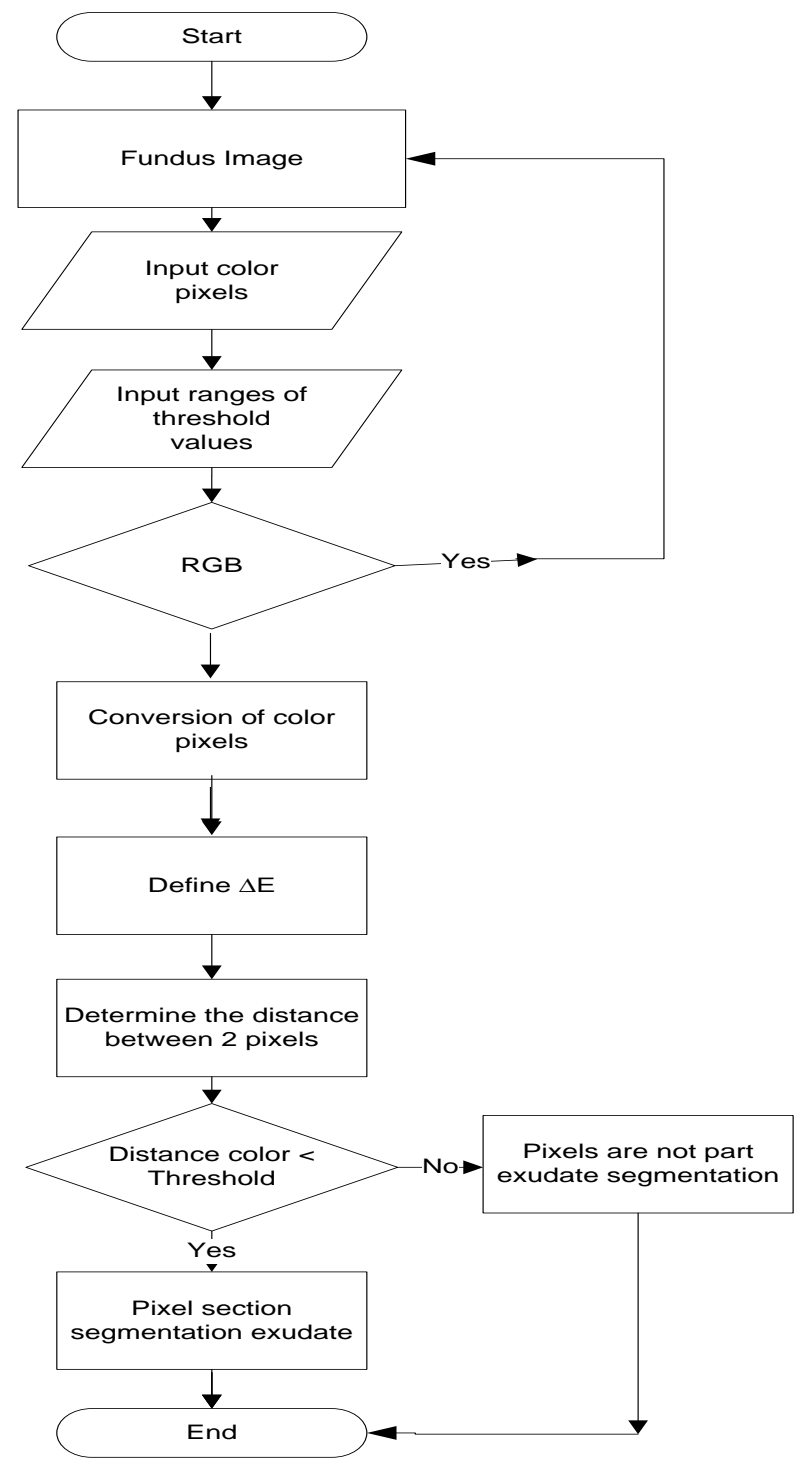

Figure 1. General Chart for Determining ROI Exudates

\subsection{Color Space Conversion Process}

Exudates segmentation consists of some segment:

1. RGB color space and Euclidian distance formula.

2. HSV space and distance $D_{\text {cyl }}$ with equation (3)[8]

$$
D_{c y l}=\sqrt{\left(L_{1}-L_{2}\right)^{2}+\left(C_{1}\right)^{2}+\left(C_{2}\right)^{2}-2 C_{1} C_{2} \cos \left(H_{1}-H_{2}\right)}
$$

3. HCL space and distance $\mathrm{D}_{\mathrm{HCL}}$ with equation (4) [8]

$$
D_{H C L}=\sqrt{\left.A_{L}\left(L_{1}-L_{2}\right)^{2}+A_{C H}\left(C_{1}+C_{2}\right)^{2}-2 C_{1} C_{2} \cos \left(H_{1}-H_{2}\right)\right)}
$$

The steps to transform RGB to HSV for detection of exudates in color fundus of retinal images can be seen below:

1. Read the RGB fundus image 
2. Transpose RGB image to HSV image using MATLAB. The pseudocode for transposing the image is:

$$
\begin{aligned}
& \text { image }=\operatorname{rgb} 2 \mathrm{hsv}(\text { rgbImage }) ; \\
& \mathrm{h}=\text { image }(:,:, 1) ; \\
& \mathrm{s}=\text { image }(:,:, 2) ; \\
& \mathrm{v}=\text { image }(:,,:, 3) ;
\end{aligned}
$$

3. Performing image filter based on the reference values (for determining the value of $T$ ) and as a tolerance value with $x$ as HSV color on the existing pixel.

4. If the colors are not include in the range of $T$, they are colored with black.

5. Re-transpose the RGB image

6. Display the color filter results ranging from $T$ until $x$ $+T$ where $x$ is the color references (exudates).

In general, the RGB color space conversion process using Euclidian distance equation can be seen in Figure 2.

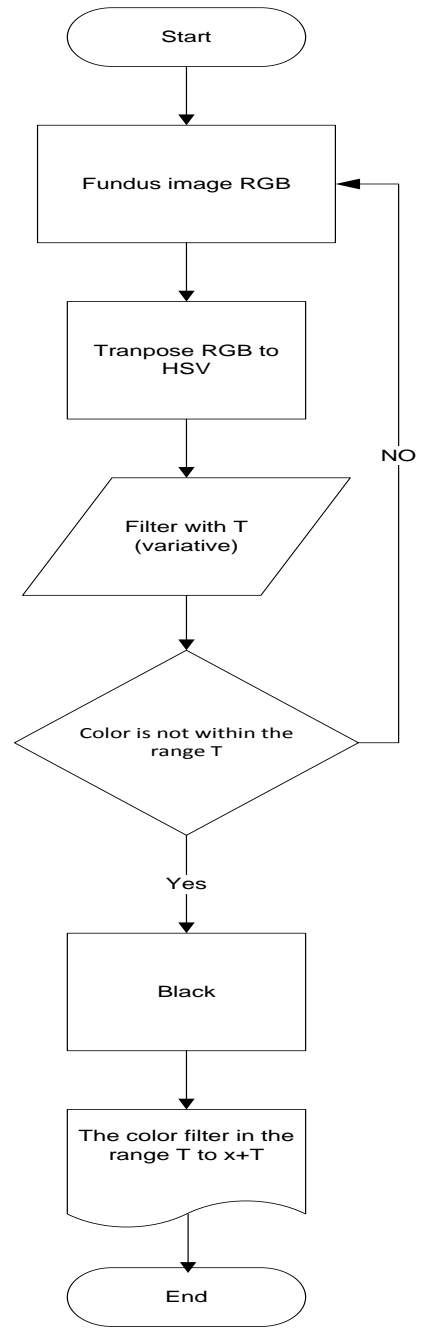

Figure 2. General Chart of RGB Color Space Conversion Process

\subsection{Optic Disc Eliminating Process}

In this experiment, the color reference of value $x$ is determined with some tolerance value $T$ to specify the similarity level of exudates color to be segmented. There are some difficulties in exudates detection since exudates have almost similar color intensity with optical disc as optic disc is a part of retinal image object. The pixel in optic disc is not required and must be removed since this optic disc is considered as noise. The experiments in this paper tag the connected component to remove pixel optic disc that have color intensity similarity with the exudates. The tagging is carried on by examining pixel $(P)$ by pixel (from left to right and top to down) to identify the area of connected pixel (conn). Conn is an area from the adjacent pixel that have similar intensity or the intensity values are within the set of $V$ (In binary image $V=\{1\}$ ). Component connectivity is linked to the input image with connectivity rules $(4,8$ or $\mathrm{m}$ connectivity). The standard connectivity for image fundus $2 \mathrm{D}$ is 8-pixels. The component marking connection for 8connected is similar to 4-connected. Only the neighboring pixels are examined; beside left and right pixels, the two upper diagonal pixels of $P$ also examined. Therefore, there are 4 neighboring pixels $P$ that are tested. They are upper, left, left diagonal and right diagonal pixel [9] as can be seen in Figure 3 .

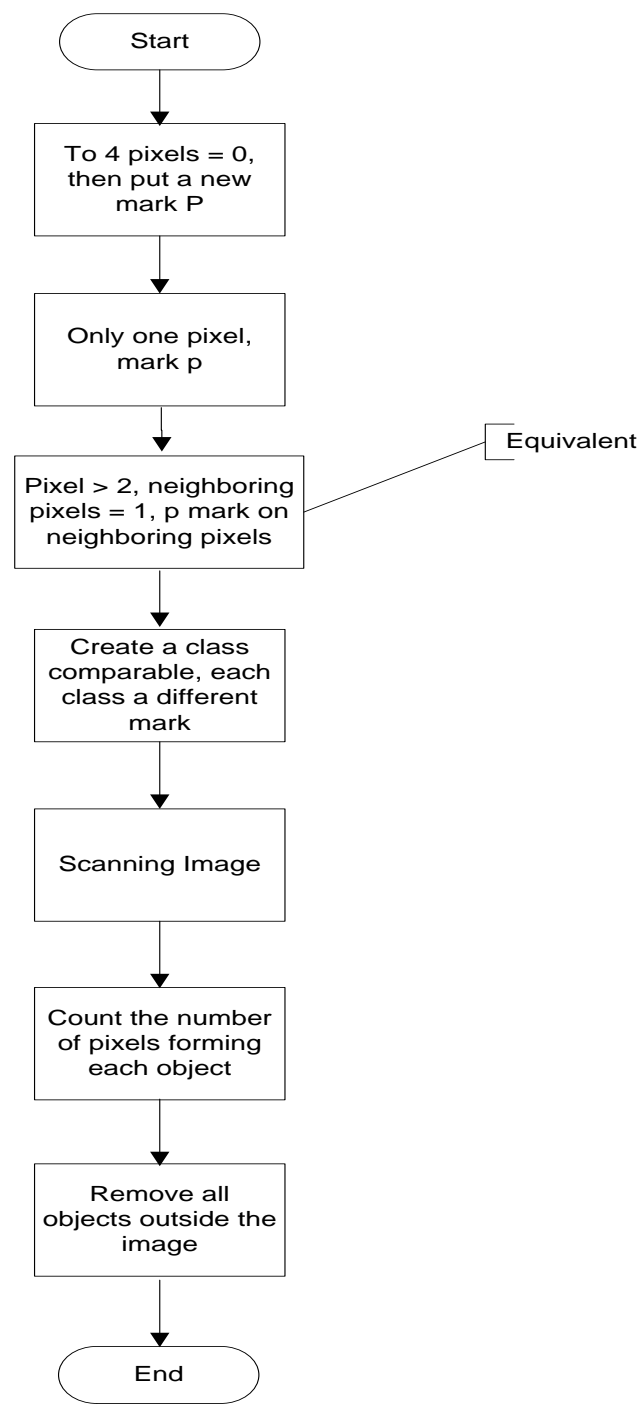

Figure 3. Optic Disc Eliminating Process 
Table 1. The Experiment Results of Exudates Segmentation

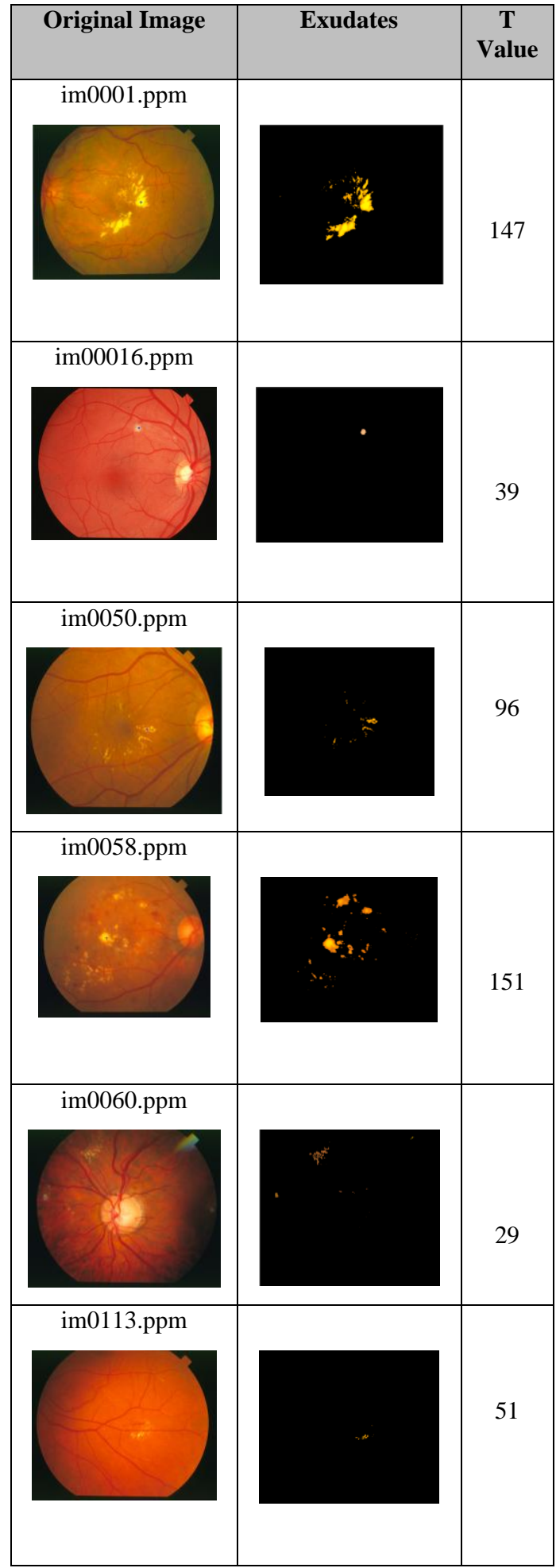

\section{RESULT AND DISCUSSION}

In general, the experiment of segmentation based on color space approach in this paper can be seen in Figure 5. During the pseudocode execution, the operator should click twice on the same image in the area of the exudates image that going to be segmented. The RGB color of the image pixel fundus is used as references to measure the color distance in RGB color space. In this paper, the experiment is carried out using image fundus with exudates according to some reference of $\mathrm{T}$ values.

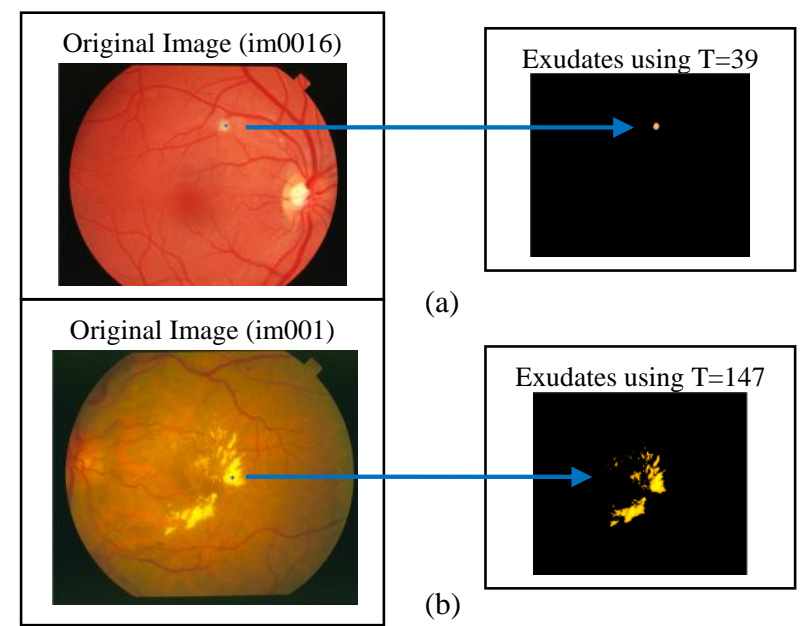

Figure 4. Exudates Segmentation with Varied Values of $\boldsymbol{T}$. (a). $T=29$. (b) $T=147$

There are many researches working on the area of exudates detection. The previous research use exudates segmentation based on green channel approach. The drawback of this approach is the misinterpretation in segmentation process between exudates and optic disc in retinal fundus image. Both of these objects have similarity of color intensity. Our approach overcomes this drawback by proposing exudates detection based on color references. The exudates are perfectly segmented without the part of optic disc. Based on the test results in Table 2 are used to determine the sensitivity, specificity and accuracy of the system based on the equation presented [10].

Table 2. Sensitivity, Specificity and Accuracy Based on Color Reference Approach

\begin{tabular}{|c|c|c|}
\hline $\begin{array}{c}\text { Sensitivity } \\
\text { in \% }\end{array}$ & $\begin{array}{c}\text { Specificity } \\
\text { in \% }\end{array}$ & $\begin{array}{c}\text { Accuracy } \\
\text { in \% }\end{array}$ \\
\hline $96.87 \%$ & $78 \%$ & $95.54 \%$ \\
\hline
\end{tabular}

Table 2 shows the results of sensitivity, specificity, and the percentage of accuracy for the trial image. The test results show that the system can detect exudates to the sensitivity of the system reached $96.87 \%$ and specificity of the system reaches $78 \%$. The accuracy of the system is $95.54 \%$. Based on the results in Table 2 clearly shows that the detection of exudates based on reference color space approach provides excellent results with the accuracy of the system (Table 2) compared with the accuracy based on green channel approach. The detection of the reference color space based exudates contributes significantly to increasing the detection accuracy of exudates of $22.01 \%$ (Figure 5). 


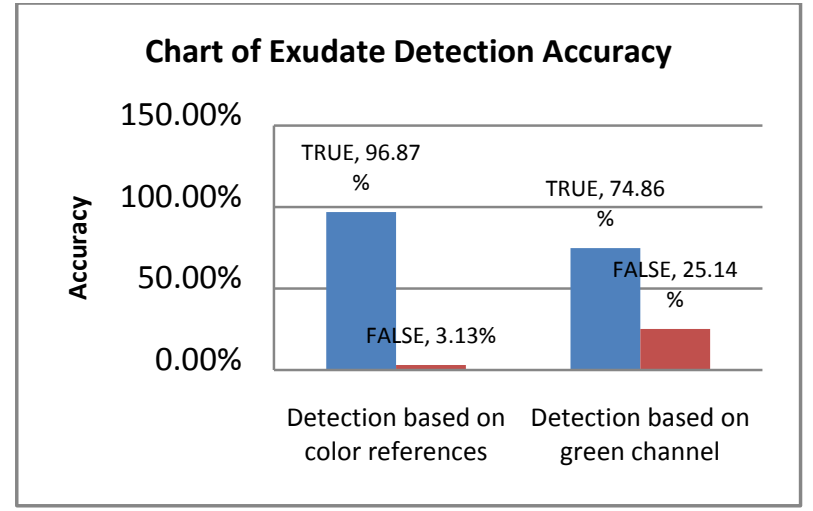

Figure 5. Chart of Exudates Detection based on Color Reference Approach and Green Channel Approach

\section{CONCLUSION}

Based on the obtained results, our experiments applied varied threshold values which are the limit boundary of the best exudates color similarity that are used for segmentation process according to the color. The value of $T$ can be customized to the preferred level of precession similarity. The higher the threshold value used the harder the exudates, otherwise decrease the value of $T$ for soft exudates (e.g., cotton wool spot). The obtained accuracy value for exudates detection using color references space is $95.54 \%$ using ground truth data of image comparison according to the color of the exudates area. This accuracy is achieved by comparing the pixels types of the exudates that are correctly detected and not. For future works, the number of extracted pixels and the establishment of exudates area are calculated. The counted pixels then multiplied by the size of the pixels according to the resolution of image acquisition.

\section{ACKNOWLEDGMENTS}

We are indebted to the experts who have contributed towards development of the template.The authors would like to acknowledge to Gunadarma University, dr Rakhma Indria Hapsari.,SpA from Awal Bros Hospital Tangerang (Ophtamologist), Research Center of Medical-Informatics of Gunadarma University

\section{REFERENCES}

[1] Vaughan DG, Asbury T, Riordan Eva P. General Ophthalmology. Edisi 17. London: McGraw-Hill. (2007)
[2] Jack J. Kanski, Brad Bowling. Clinical Ophthalmology: A Systematic Approach 7th Edition. Butterworth Heinemann Elsevier. ISBN-13: 978-0702040931. (2011).

[3] B.Ramasubramanian and G.Mahendran. An Efficient Integrated Approach for the Detection of Exudates and Diabetic Maculopathy in Colour fundus Images. Advanced Computing: An International Journal ( ACIJ ), Vol.3, No.5. (2012)

[4] Joussen A.M.2007. Retinal Vascular Diseease. New York: Springer. p. 3-5, 66-70, 129-132, ,228-31, 309, 291-331

[5] Nidhal K. El Abbadi, Enas Hamood Al-Saadi. Automatic Detection of Exudates in Retinal Images. International Journal of Computer Science Issues Vol.10, Issue 2 No.1. ISSN (Print): 1694-0814 | ISSN (Online) : 16940784.(2013)

[6] Marwan D. Saleh, C.Eswaran and Ahmed Mueen. (2011). An Automated Blood Vessel Segmentation Algorithm Using Histogram Equalization and Automatic Threshold Selection. Journal of Digital Imaging.DOI: $10.1007 / \mathrm{s} 10278-010=9302-9$.

[7] Ratna Bhargavi and V. Rajesh. (2015). Detection And Feature Extraction Using Active Contour Model And Sift In Color Fundus Images. ARPN Journal of Engineering and Applied Sciences. VOL. 10, NO. 6.

[8] Sarifuddin Madenda. Pengolahan Citra \& Video Digital. Teori, Aplikasi dan Pemrograman Menggunakan Matlab. Ed. Erlangga.ISBN. 978-602-298-598-3. (2015)

[9] Rodiah, Sarifuddin Madenda, Fitrianingsih. ThreeDimensional (3D) Reconstruction for Detectiong Shape and Volume of Lung Cancer Nodules. IPTEK Journal ITS. No.1. (2014).

[10] Priya, R., Aruna, P., Review of Automated Diagnosis Of Diabetic Retinopathy using The Support Vector Machine, International Journal of Applied Engineering Research, No. 4, Vol. 1, pp: 844-863. 2010

[11] Gonzalez R. C. and Woods E. E., Digital Image Processing. $3^{\text {rd }}$ Edition. Prentice Hal;. (2007) 